\title{
Night-time light derived estimation of spatio-temporal characteristics of urbanization dynamics using DMSP/OLS satellite data
}

\author{
Ting Ma ${ }^{\text {a,* }}$, Yuke Zhou ${ }^{\text {a }}$, Chenghu Zhou ${ }^{\text {a }}$, Susan Haynie ${ }^{\mathrm{b}}$, Tao Pei ${ }^{\mathrm{a}}$, Tao Xu ${ }^{\mathrm{a}, \mathrm{c}}$

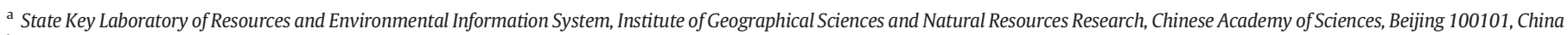 \\ ${ }^{\mathrm{b}}$ Demographic Consulting, Inc., Santa Ana, CA 92706, USA \\ c University of Chinese Academy of Sciences, Beijing 100049, China
}

\section{A R T I C L E I N F O}

\section{Article history:}

Received 20 May 2014

Received in revised form 11 October 2014

Accepted 16 November 2014

Available online 11 December 2014

\section{Keywords:}

Night-time light

Urbanization

DMSP/OLS

Quadratic relationship

China's cities

\begin{abstract}
A B S T R A C T
Understanding the spatio-temporal dynamics of urban development at regional and global scales is increasingly important for urban planning, policy decision making and resource use and conservation. Continuous satellite derived observations of anthropogenic lighting signal at night provide consistent and efficient proxy measures of demographic and socioeconomic dynamics in the urbanization process. Previous studies have demonstrated significant positive correlations between the nocturnal light brightness, mainly derived from the Defense Meteorological Satellite Program/Operational Linescan System (DMSP/OLS), and population and economic variables. Quantitative measurements of artificial lighting emissions at night therefore can be indicative of the overall degree of socioeconomic development at regional to country levels. The spatio-temporal characteristics of anthropogenic night-time lighting, potentially connected to the dynamic patterns of spatially expanding human settlement and economic activities during the urban expansion process, however, has received less attention largely because of diversity of both socioeconomic activity and urban forms. Based upon the quadratic relationship between the pixel-level night-time light radiance and corresponding brightness gradient (i.e. the rate of maximum local change) at the local scale, we here proposed a spatially explicit approach for partitioning DMSP/OLS night-time light images into five types of night-time lighting areas for individual cities: low, medium-low, medium, medium-high and high, generally associated with urban sub-areas experienced distinctly different forms and human activity. At the country scale, our findings suggest that significant rises are commonly found in these five types of night-time lighting areas with different growth rates across 271 China's cities from 1992 to 2012 . At the urban scale, however, five types of night-time lighting areas show various trends for individual cities in relation to the urban size and development levels. The marked increase in high night-time lighting area is highly prevalent in most of China's cities with rapid urbanization over the past 21 years while significantly decreased low and medium-low night-time lighting areas are most likely to occur in large and extra-large cities. Moreover, the transition between different types of night-time lighting areas could further portray the spatiotemporal characteristics of urban development. Analyzing results indicate that the spatial expansions of gradually intensified night-time light brightness correspond geographically with the rural-urban gradients following a stepwise transition of night-time light brightness during the urban expansion.
\end{abstract}

(c) 2014 Elsevier Inc. All rights reserved.

\section{Introduction}

Urbanization is a complicated phenomenon involving increasing populations, escalated socioeconomic activity and spatially expanded builtup area and infrastructures (Cohen, 2006; Montgomery, 2008; Pugh, 2000). Measurement of spatio-temporal characteristics of urban development is essential for understanding the environmental consequences of

\footnotetext{
* Corresponding author. Tel.: + 861064889769 .

E-mail address: mting@lreis.ac.cn (T. Ma).
}

anthropogenic resource consumption and landscape change in the context of environment and resource sustainability (Folke, Jansson, Larsson, \& Costanza, 1997; Johnson, 2001; Shukla \& Parikh, 1992). Long term archives of satellite based observations of anthropogenic night-time brightness provide an efficient way to continuously monitor recent human activity and socioeconomic dynamics during urban processes in a changing world (Croft, 1973; Doll, Muller, \& Elvidge, 2000; Elvidge, Baugh, Kihn, Kroehl, \& Davis, 1997; Small, Pozzi, \& Elvidge, 2005; Sutton, 2003). In comparison with other satellite products mainly focusing on mapping land cover and land use, a salient feature of nighttime light data is that remotely sensed changes in anthropogenic nighttime lighting signals can be quantitatively connected to simultaneous 
alterations in demographics, the economy, energy consumption and urban extent (Elvidge et al., 2009; Ghosh et al., 2010; Imhoff, Lawrence, Stutzer, \& Elvidge, 1997). Satellite-derived night-time light data therefore have been extensively used as observationally based metrics for estimating human development and urbanization processes (Elvidge et al., 2001; Ghosh, Anderson, Elvidge, \& Sutton, 2013; Small and Elvidge, 2011; Sutton \& Costanza, 2002; Sutton, Roberts, Elvidge, \& Baugh, 2001).

Night-time light imagery, derived from the Defense Meteorological Satellite Program/Operational Linescan System (DMSP/OLS), excluding glare and sunlit effects, provide spatially explicit observations of artificial lighting sources across human settlements at night without moonlight (Elvidge et al., 1999). A number of efforts have been made to successfully capture the robust positive correlations between DMSP/ OLS night-time light data and demographic and socioeconomic variables at regional to global scales (Chen \& Nordhaus, 2011; Doll, Muller, \& Morley, 2006; Elvidge, Baugh, Anderson, Sutton, \& Ghosh, 2012; Small et al., 2005; Sutton et al., 2001; Zhang \& Seto, 2011). Most previous studies have attempted to use the overall measures of regional night-time lights for estimating corresponding demographic and socioeconomic activities (Doll et al., 2006; Elvidge et al., 2001; Ghosh et al., 2010; Sutton et al., 2001). The quantitative connections between night-time light brightness and urbanization parameters for a given target region are usually constructed through counting the area covered by lit pixels (i.e. the area of lighting) or summing total radiances of pixel light (i.e. the sum of night-time radiance) exceeding the pre-chosen threshold. Both these indices enable us to examine overall responses of night-time light to regional socioeconomic activity over time and to compare the interregional differences of urban development (Doll et al., 2006; Elvidge et al., 2001; Ghosh et al., 2010; Zhang \& Seto, 2011). Intraregional spatial variations of night-time light signals, which are likely correlated with the fluctuations of spatial patterns of urbanization dynamics at a local scale, however, have received less attention. Limited understanding of spatio-temporal evolutions of artificial night-time light brightness hampers the further applications of DMSP/OLS data in urban studies, particularly for investigating temporal evolutions of interior development patterns of individual cities.

There are well-documented weaknesses regarding DMSP/OLS nighttime light data: (1) the effects of over-glow and shrink of night-time light can often lead to overestimates or underestimates of the spatial extent of urban land (Elvidge et al., 1997; Imhoff et al., 1997), and (2) no empirical brightness threshold is widely applicable for extracting the lit area precisely matching the actual boundary of urbanized areas owing to various urbanization patterns (Small, Elvidge, Balk, \& Montgomery, 2011; Small et al., 2005). These two practical drawbacks likely constitute challenges for partitioning and classifying night-time light images in association with different degrees of human activity over space. Consequently, it becomes difficult to portray the spatio-temporal patterns in anthropogenic night-time lighting signals in relation to the spatial dynamics of socioeconomic activity over time during urban development owing to the lack of available measures of spatial fluctuations and temporal transitions of night-time lights at a local scale. An improved approach to outline the local spatio-temporal features of anthropogenic night-time brightness therefore is highly needed for studying changes in interior spatial patterns of urban processes using night-time light based observation data.

The primary objective of this study is to quantitatively estimate spatio-temporal dynamics of urban development for China's cities by analyzing spatial changes in anthropogenic night-time light signals over time across human settlements at a local scale. Firstly, we develop a quantitative partitioning approach for DMSP/OLS nighttime light imagery to identify different types of urban sub-regions with various magnitudes of artificial nocturnal lighting based upon the quadratic relationship between the pixel-level night-time light brightness and corresponding spatial gradient of night-time light signals. Finally, spatio-temporal characteristics of urbanization dynamics across all China's prefectural level cities and municipalities from 1992 to 2012 are carried out through investigating long-term dynamics and inter-annual transitions in different types of urban sub-regions derived from DMSP/OLS night-time light data.

\section{Data and methods}

\subsection{Long-term datasets of DMSP/OLS night-time lights}

Time series data of DMSP/OLS stable night-time light imagery spanning years 1992-2012 were derived from NOAA's National Geophysical Data Center (version 4, downloaded from the website of http://ngdc. noaa.gov/eog/dmsp.html on March-10-2014). These image products provide gridded cell based annual cloud-free composited stable nighttime lights with a digital number (DN) ranged from 0 to 63. DMSP/ OLS night-time light data from 1992 to 2012 were obtained by six individual sensors: F10 (1992-1994), F12 (1994-1999), F14 (1997-2003), F15 (2000-2007), F16 (2004-2009) and F18 (2010-2012). Following Elvidge et al. (2009, 2013), we employed the second order regression model with respect to the composite of F12 in 1999 to empirically inter-calibrate DMSP/OLS night-time light images in order to eliminate inter-annual variations and response differences among sensors. Moreover, for the year with two annual composites collected by two separate sensors, the night-time light image exhibiting the best-fitting regression in inter-calibration was chosen in our study. We then initially extracted the time series of night-time light data from 1992 to 2012 for 285 China's prefectural level cities and municipalities by overlapping the administrative boundary map onto the processed DMSP/OLS night-time light images with the spatial resolution of 30 arc-seconds under the WGS84 coordinate reference system.

\subsection{The spatial gradient of night-time lights}

To depict the pixel-level fluctuations of night-time light (NTL) across human settlements, we used the brightness gradient $(B G)$ to measure spatial changes in artificial night-time lighting signals. As shown in Fig. 1(a), $B G$ is defined as the rate of maximum change in NTL from the site to its neighbors (rise) across the corresponding geographical span (run). Thus, the output $B G$ can be calculated as:

$B G=\frac{\text { rise }}{\text { run }}$

It should be noted that the range of output $B G$ values depends on the type of measurement units. For DMSP/OLS night-time light imagery, if we set the value of run to the pixel size ( $\sim 0.00833$ decimal degree), a small range of output $B G$ (expect rise $=0$ ) is yielded from 89.52 (in degrees, when rise $=1$ ) to 89.99 (when rise $=63$ ). To increase comparability between rise in NTL and run in geographical span for the pixel-level $B G$ calculation, the value of run is set to 63 to match the saturates NTL value. Thus, theoretical maximum and minimum values (expect rise $=0$ ) for $B G$ at the pixel level are 45.00 and 0.91 when NTL rise $=63$ and rise $=$ 1 , respectively.

For gridded night-time light images, the pixel-level $B G$ is regarded as a measure of the maximum change in NTL over one pixel size between the grid cell and its neighbor cells. In this study, the average maximum technique (Burrough \& McDonell, 1998) was applied to calculate BG for each grid cell. This algorithm applies weighting coefficients which are proportional to the reciprocal of the square of the distance from the processing grid cell for the nearer NTL values. Because we mainly focus on the pixel-level changes in NTL over space, eight neighbor cells of the processing cell were taken account into in the calculation of $B G$. As shown in Fig. 1(b), the pixel-level $B G$ can hence be estimated by the rates of change of NTL in the horizontal $(d N T L / d x)$ and vertical 

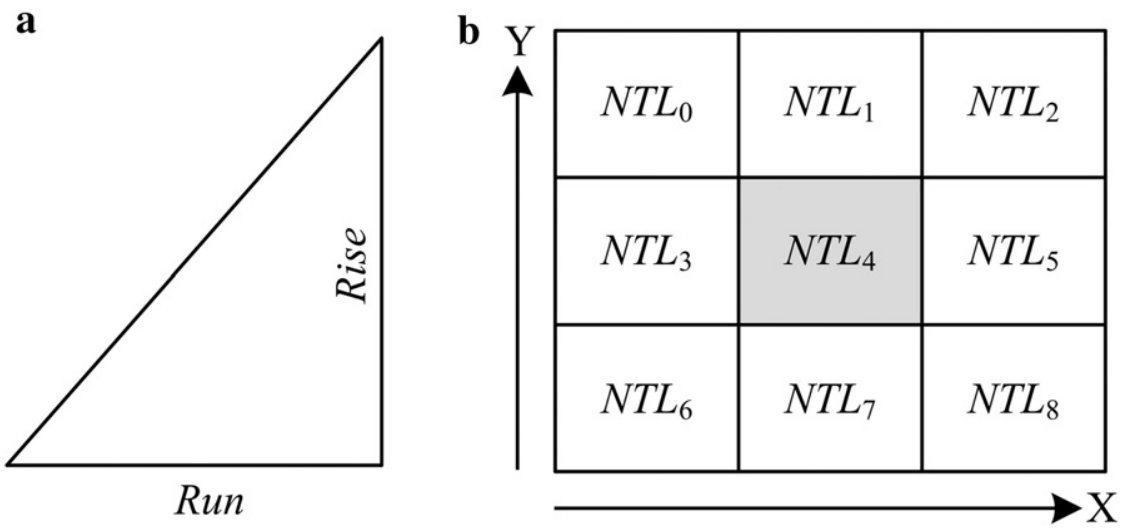

Fig. 1. Schematic diagram of the definition a) and calculation b) of the brightness gradient.

( $d N T L / d y)$ directions from the central grid cell to its eight adjacent grid cells as following:

$B G=\sqrt{d N T L / d x^{2}+d N T L / d y^{2}}$

$[d N T L / d x]=\left[\left(N T L_{2}+2 N T L_{5}+N T L_{8}\right)-\left(N T L_{0}+2 N T L_{3}+N T L_{6}\right)\right] /(8 \times$ run $)$ $[d N T L / d y]=\left[\left(N T L_{6}+2 N T L_{7}+N T L_{8}\right)-\left(N T L_{0}+2 N T L_{1}+N T L_{2}\right)\right] /(8 \times$ run $)$

Moreover, to reduce the effects of distortions in distance and area under the geographic projection, we applied the great circle distance and the ellipsoid trapezoid area to calculate the size and the area of NTL pixels in the following analyses.

\subsection{The relationship between night-time lights and the brightness gradient}

A case example in Fig. 2 illustrates the urban-scale spatial fluctuations of both the pixel-level night-time light and corresponding brightness gradient across Huaian City in 2012. Comparisons with a high resolution satellite image and land cover maps with ancillary geographic data qualitatively reveal that grid cells with low $B G$ values are likely to be found in both the central region of the urban area with intensified human activity and high NTL and the rural region with less human activity and low NTL. Grid cells with high $B G$ values commonly appear in urban-rural transition zone with medium NTL. As shown in Fig. 3(a) and (b), we could further find that the quantitative relationship between $B G$ and NTL is not single valued for cities that are not small, isolated and circular and there is a wide range of brightness gradients associated with all but the brightest and dimmest pixels. Therefore, the relationship between the pixel-level NTL and BG for a given city can be fitted by a quadratic polynomial:

$B G=a N T L^{2}+b N T L+c$

where $a, b$ and $c$ represent fitting coefficients. In order to examine the prevalence of this statistical relationship, we performed quadratic regressions for the pixel-level NTL and $B G$ at a local urban scale using the least squares method for each of 285 Chinese cities and 21 annual time periods (1992 through 2012). The quadratic regression results show that only 21 of 5985 quadratic regressions were rejected by $F$-test at the $5 \%$ significance level. This result states that the quadratic relationship between NTL and BG is likely to prevail at the local scale regardless of variations in satellite sensors, urban size and urban forms.

\subsection{Spatial partition of night-time light imagery}

The robust quadratic relationships between the pixel-level NTL and $B G$ across most of Chinese cities allow us to quantitatively classify the urban area according to conspicuous differences in anthropogenic nocturnal lights at a local scale. Based upon the graph of the fitted quadratic polynomial (a downward parabola with coefficient $a<0$, see Fig. 3(c)), we here spatially subdivided a city into five different sub-regions involving: low (night-time lights range from $N T L_{0}$ to $N T L_{1}$ ), medium-low (from $N T L_{1}$ to $N T L_{2}$ ), medium (from $N T L_{2}$ to $N T L_{3}$ ), medium-high (from $N T L_{3}$ to $N T L_{4}$ ) and high (from $N T L_{4}$ to $N T L_{5}$ ) night-time lighting areas. As shown in Fig. 3(c), the split point $P_{2}$ is the turning point of the parabola in which the maximum $B G$ and medium NTL, typically occurring in the transition zone of urban area and suburban, are observed. We then set other split points based upon $P_{2}, P_{0}$ (with the minimum $N T L$ ) and $P_{5}$ (with the maximum NTL). The $B G$ value of the split point $P_{1}\left(P_{3}\right)$ is the average of $B G$ values of $P_{0}$ and $P_{2}\left(P_{2}\right.$ and $\left.P_{5}\right)$. The $B G$ value of $P_{4}$ is the average of $B G$ values of $P_{3}$ and $P_{5}$. The detailed calculations for partitioning intervals (from $N T L_{0}$ to $N T L_{5}$ ) and corresponding $B G$ values are presented in Table 1 . This algorithm implies that for a given city with significant quadratic relationship between pixel-level night-time light and spatial brightness gradient, the partition intervals for night-time light imagery are determined by the range of NTL and the fitted coefficients of the quadratic function. This also means that no empirical threshold and parameterization of the split points are required for partitioning night-time light imagery across different cities. Moreover, the primary advantage of quadratic curve based partitioning method for DMSP/OLS night-time light imagery is that it reduces the impacts of various urban development patterns because the partition method is based upon the relative quantitative relationship between the pixel-level NTL and BG at a local scale.

Fig. 3(d) displays the spatially partitioned result of DMSP/OLS nighttime light image for Huaian City in 2012. From this figure, we see that (1) the highly lighted region (with $\mathrm{DN}>56$ in this case) likely represents the developed area of the urban land with intense human activity and high percent of impervious surface, (2) the medium-highly lit region (with DN range of 51-56) appears to be linked to the periphery of the central area with considerable human activity, (3) the medium nighttime lighting region (with DN range of 33-50) likely covers the suburban area, (4) the medium-low night-time lighting region (with DN range of 13-32) mainly contains the rural-urban transition zone and town level residential places, and (5) low night-time lighting region (with $\mathrm{DN}<12$, the same observation was obtained by Small et al. (2011)) primarily contains agricultural sites and small villages with low human activity.

As exemplified by Tianjin City in 2012 (Fig. 3(e)-(i)), a extra-large city with more complicated urban forms and spatial distributions of socioeconomic activity than Huaian City, the quadratic relationship 

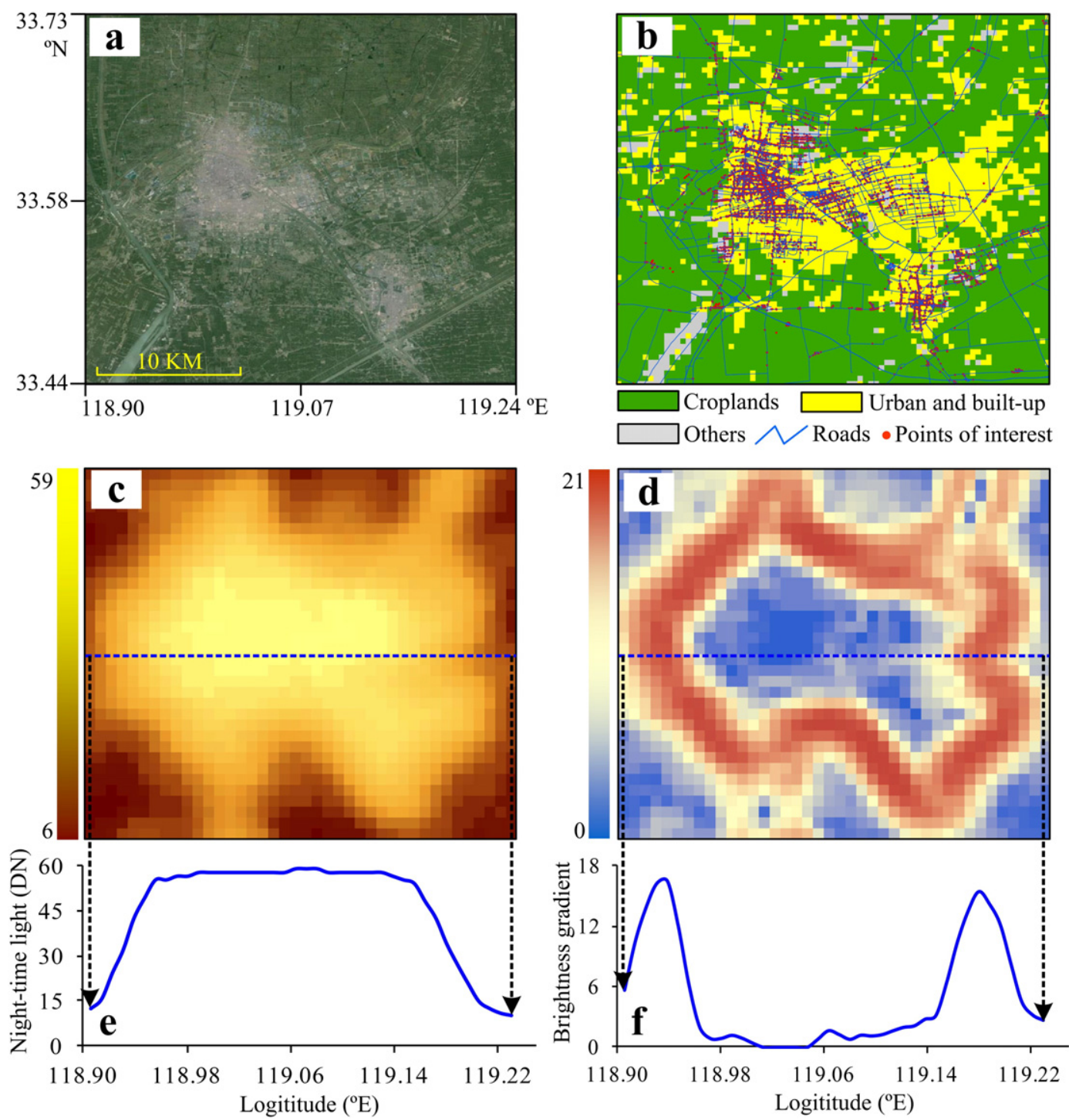

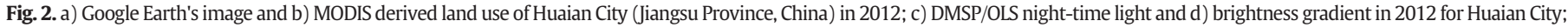
e) and f) show profiles of the pixel-level night-time light and brightness gradient in the latitudinal transect ( $\left.33.58{ }^{\circ} \mathrm{N}\right)$ across Huaian City, respectively.

between pixel-level NTL and $B G$ is statistically significant (adjusted $R^{2}=$ $0.57, P<0.001$ ) even though there exist several spatially separated areas with highly night-time lighting and intensified human activity. The classification results for night-time light imagery can be validated through comparisons with high resolution land use map and ancillary geospatial datasets (e.g. points of interest in Fig. $3(\mathrm{~g})$ ). Example comparisons in Fig. $3(\mathrm{~g})$ and (i) show spatial correspondences of high lighting areas with dense artificial surfaces as well as dim and lower night-time lighting areas with agricultural and less dense population land use, respectively. Quantitative comparisons of two classified images suggest that the sum of medium, medium-high and high night-time lighting areas account for $\sim 30 \%$ of the city area in Tianjin comparable with nearly $28 \%$ of the urban land identified as artificial surfaces derived from $30 \mathrm{~m}$ Landsat imagery. Furthermore, our analyzing results regarding the quadratic regression and comparisons of classified night-time images and land use map for all China's prefectural level cities and municipalities with various urban size and urban forms verified the prevalence of the quadratic relationship between the pixel-level NTL and BG. This also indicates that the quadratic curve based partition method for DMSP/OLS imagery is insensitive to the spatial distribution of nighttime lighting signals at a local scale.

\subsection{Spatio-temporal analysis of night-time light dynamics for China's cities}

11 of 285 of China's cities (most are small cities with low night-time light emissions in the central area), in which insignificant quadratic fittings have been observed in individual years, were excluded from the subsequent analyses. Yearly partitioned DMSP/OLS night-time light images from 1992 to 2012 for other 274 China's prefectural level cities and municipalities were yielded separately based upon the corresponding quadratic regression formulas. Long term trends in different types of night-time lighted areas at both the country level and the city level were analyzed through the maximum likelihood linear regression. The significance testing was performed by two-tailed $t$-test. The differences in long-term trends in five types of urban night-time lit areas among various cities were carried out in order to compare the diversity of urban development process in China.

Long-term series of classified DMSP/OLS night-time light images can potentially be used to characterize spatial and temporal dynamics in demographic and socioeconomic activities of urban development for individual cities through surveying local changes and transitions in different types of night-time lighting areas of urban land. In this study, we examined pixel-based transitions of night-time lighting type among three 

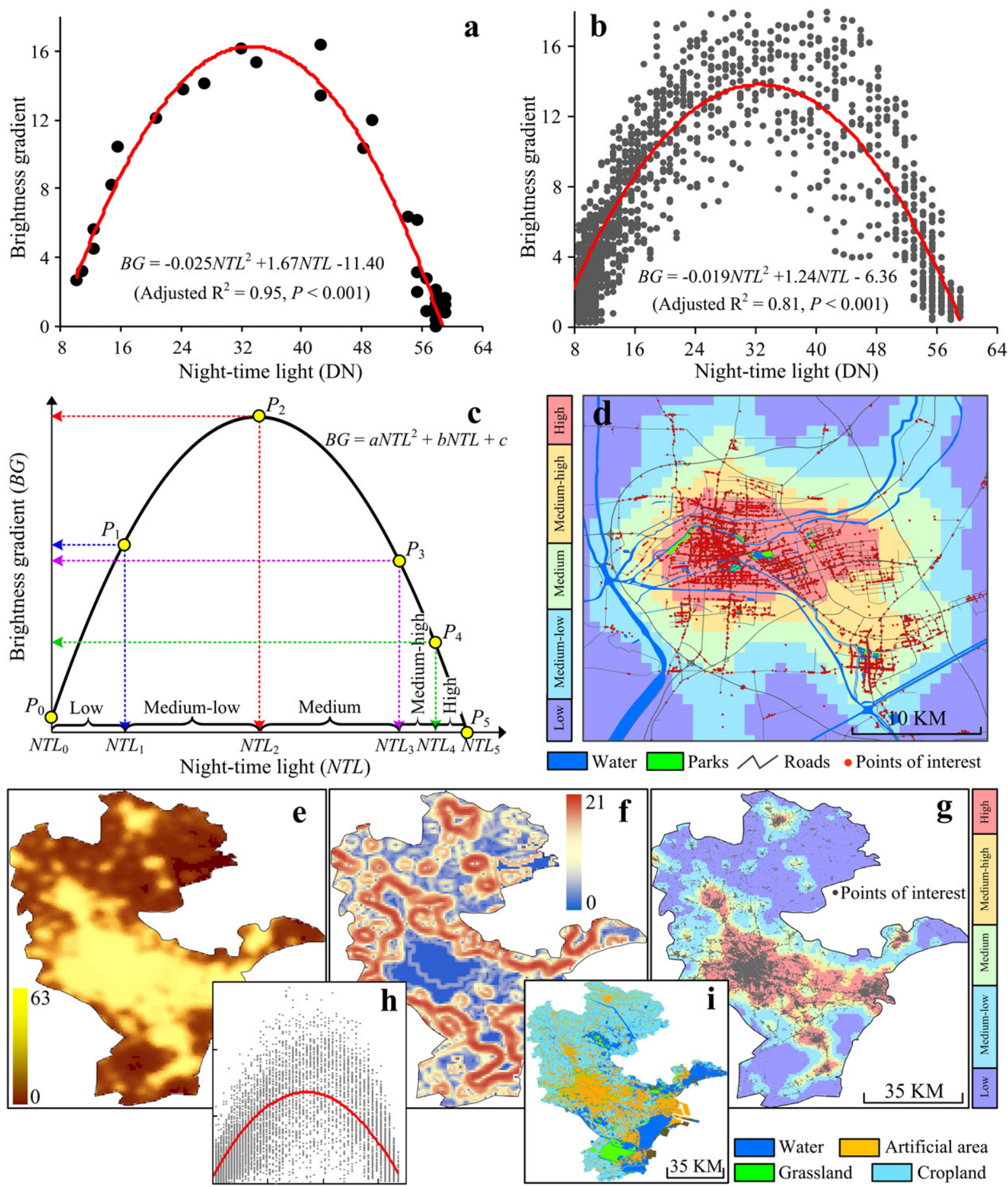

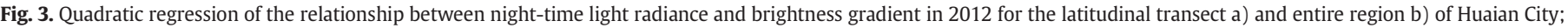

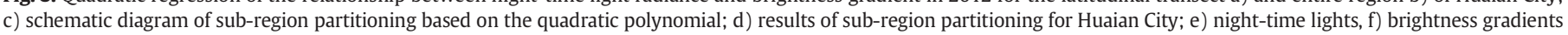

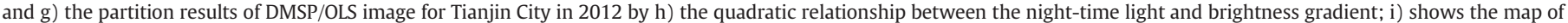
$30 \mathrm{~m}$ Landset data derived land use of Tianjin City in 2012.

years: 1992, 2002 and 2012 to obtain an estimate of the spatial dynamics in urban processes across 274 China's cities.

\section{Results and discussions}

\subsection{The relationship between lit area and urban built-up area}

Empirical threshold based night-time lighting areas are known to usually mismatch the spatial extent of developed land in the urban region due to both the over-glow effect of night-time light and the spatial heterogeneity of human activity and infrastructure development (Small et al., 2011). In this study, significant positive correlations between statistical urban built-up area and two types of DMSP/OLS derived sub-regions involving high lighting areas and sum of medium-high and high lighting areas for China's cities in 2012 have been found (Fig. 4(a)). These results commonly suggested the quantitative connections between intensely night-time lighted areas and corresponding well-developed urban land. Night-time light based observations therefore could be indicative of urbanized areas with high socioeconomic activities in absence of survey data. Moreover, our results also show 
Table 1

Calculations for the split points drawn in Fig. 3(c) based upon the quadratic polynomial.

\begin{tabular}{lll}
\hline Point & Night-time light $(N T L)$ & Brightness gradient $(B G)$ \\
\hline$P_{0}\left(N T L_{0}, B G_{0}\right)$ & $N T L_{\min }$ & $\operatorname{Max}\left[0, a N T L_{\min }^{2}+b N T L_{\min }+c\right]$ \\
$P_{1}\left(N T L_{1}, B G_{1}\right)$ & $-\frac{b}{2 a}-\sqrt{\frac{B G_{1}-c}{a}+\frac{b^{2}}{4 a^{2}}}$ & $\frac{B G_{0}+B G_{2}}{2}$ \\
$P_{2}\left(N T L_{2}, B G_{2}\right)$ & $-\frac{b}{2 a}$ & $\frac{-b^{2}+4 a c}{4 a}$ \\
$P_{3}\left(N T L_{3}, B G_{3}\right)$ & $-\frac{b}{2 a}+\sqrt{\frac{B G_{3}-c}{a}+\frac{b^{2}}{4 a^{2}}}$ & $\frac{B G_{5}+B G_{2}}{2}$ \\
$P_{4}\left(N T L_{4}, B G_{4}\right)$ & $-\frac{b}{2 a}+\sqrt{\frac{B G_{4}-c}{a}+\frac{b^{2}}{4 a^{2}}}$ & $\frac{3 B G_{5}+B G_{2}}{4}$ \\
$P_{5}\left(N T L_{5}, B G_{5}\right)$ & $N T L_{\max }$ & $\operatorname{Max}_{0}\left[0, a N T L_{\max }^{2}+b N T L_{\max }+c\right]$ \\
\hline
\end{tabular}

variations in partitioning thresholds derived from quadratic regressions for extracting intensive night-time lighting regions within individual cities (Fig. 4(b) and (c)). Generally, extra-large and large cities have distinct higher DN thresholds than medium and small cities. Thus, brightness intervals for partitioning DMSP/OLS night-time light imagery for individual cities may vary when characterizing the spatio-temporal dynamics of urbanization at the local scale owing to differences in urban sizes and urban forms.

\subsection{Country level trends in different night-time lighting areas}

China has experienced rapid urbanization processes with notable economic growth since the 1990s. Yearly statistical data (National Bureau of Statistics of China, 1993-2013) indicate that total urban built-up areas of China's prefectural level cities and municipalities were dramatically increased to $33.61 \times 10^{3} \mathrm{KM}^{2}$ in 2011 from $12.41 \times 10^{3}$ $\mathrm{KM}^{2}$ in 1993 with a notable linear trend of $1.24 \times 10^{3} \mathrm{KM}^{2}$ per year $(P<0.001)$ (Fig. 5(a)). In contrast with limited statistical data which mainly focused on well-developed areas with intensified human activity, night light data reveal distinct differences in long term trends in five types of nocturnal lighting areas throughout China's cities. As shown in Fig. 5(b), high lighting areas show significant positive trends at a rate of $0.99 \times 10^{3} \mathrm{KM}^{2}$ per year $(P<0.001)$ during $1992-2002$, conspicuously higher than medium-high lighting areas yearly increased by $0.58 \times 10^{3}$ $\mathrm{KM}^{2}(P<0.001)$. Low and medium lighting areas increased by similar rates of $1.38 \times 10^{3}(P=0.006)$ and $1.24 \times 10^{3} \mathrm{KM}^{2}$ per year $(P<$ $0.001)$, respectively. An annual rise of $0.70 \times 10^{3} \mathrm{KM}^{2}(P<0.001)$ is found in medium-low night-time lighting areas. Moreover, high and medium-high lighting areas jointly experience a distinctly high growth by $1.57 \times 10^{3} \mathrm{KM}^{2}$ per year $(P<0.001)$, comparable to that of statistical urban built-up areas. These results commonly exhibit a marked urbanization process and rapid development of human settlement from 1992 to 2012. Variances among rising trends in five types of night-time lighting areas at the country level would further demonstrate relatively different increases in urban sub-regions with various demographic and socioeconomic activities during urban development across China's cities over the past 21 years. Developed urban area with intensified human activity show a faster growth than suburban and rural-urban transition areas with moderate human activity.

\subsection{Urban scale changes in different night-time lighting areas}

Country level analyses portray significant linear trends towards simultaneously increased overall spatial extents for different types of night-time lighted regions of the urban land. Statistical results at the urban scale, however, reveal diverse changes in five types of nighttime lighting areas from 1992 to 2012 among 274 China's cities. As presented in Fig. 6, 39\% of cities (mostly are small and medium cities) show an increase in low night-time lighting areas, in contrast to $35 \%$ of cities (mostly large and extra-large cities) in which distinctly reduced low night-time lighting areas are found. For medium-low lighting areas, significant positive and negative tendencies are observed in $66 \%$ and $18 \%$ of cities, respectively. The proportion of cities with distinctly enhanced medium lighting areas rises to $88 \%$ while only $4 \%$ of cities show conspicuously reduced medium lighting areas in the urban land. Furthermore, more than $90 \%$ of cities exhibit significant trends towards increase in both medium-high and medium lighting areas. These results indicate that (1) notable escalations in locally intense night-time lighting areas (including high and medium-high) are prevailing throughout 274 Chinese cities, (2) the proportion of cities with a significant positive trend in lit areas gradually increases from the type of low night-time emissions to the type of high night-time emissions, and (3) meanwhile the proportion of cities with significant positive trend in lit areas markedly decreases.

For individual cities, long-term trends in five types of night-time lighting areas might vary with urban size, urban form and development stage. As shown in Fig. 7, for instance, Xinyan City, Harbin City and Nanning City appear to have simultaneous enhancements in five types of night-time lighting areas in which low lighting areas show distinctly higher growth rate than other types with close growth rates. This likely indicates a balanced urbanization in these medium-large size cities with moderate increases in built-up areas and suburban regions. In
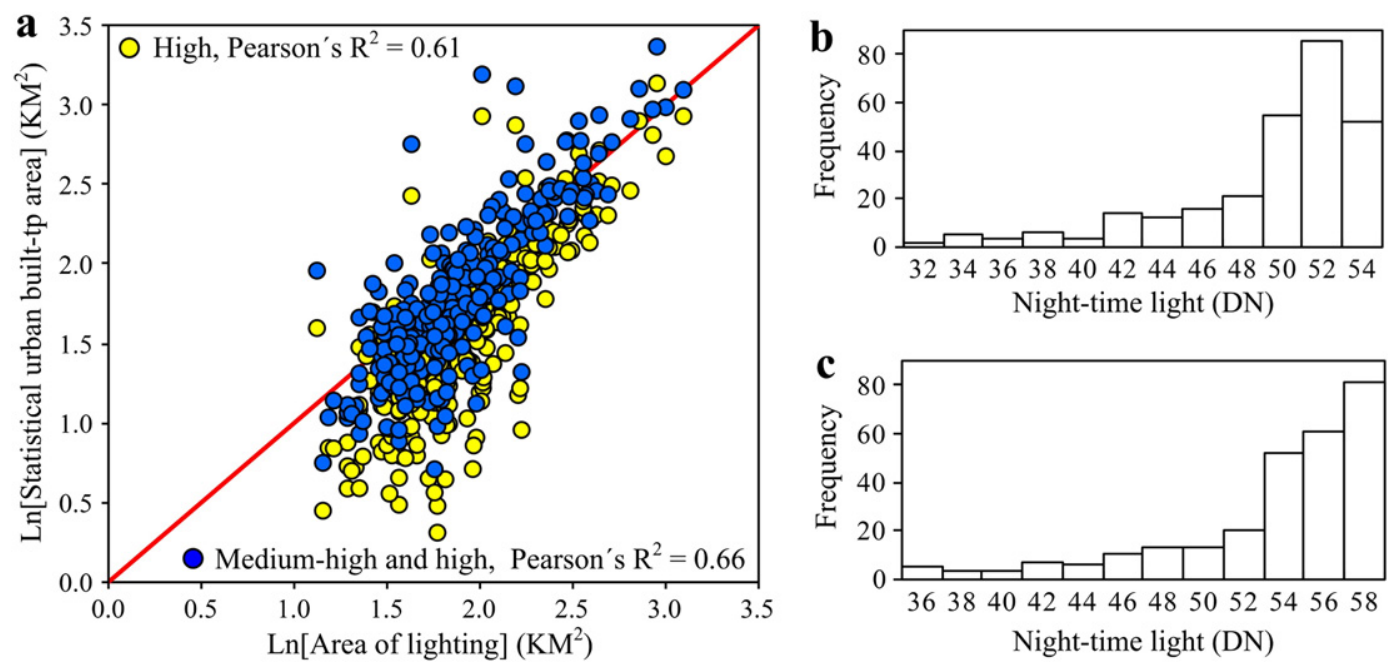

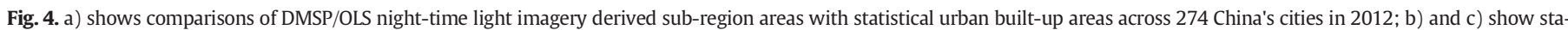
tistical histogram of night-time light thresholds for medium-high and high lighting areas, respectively. 

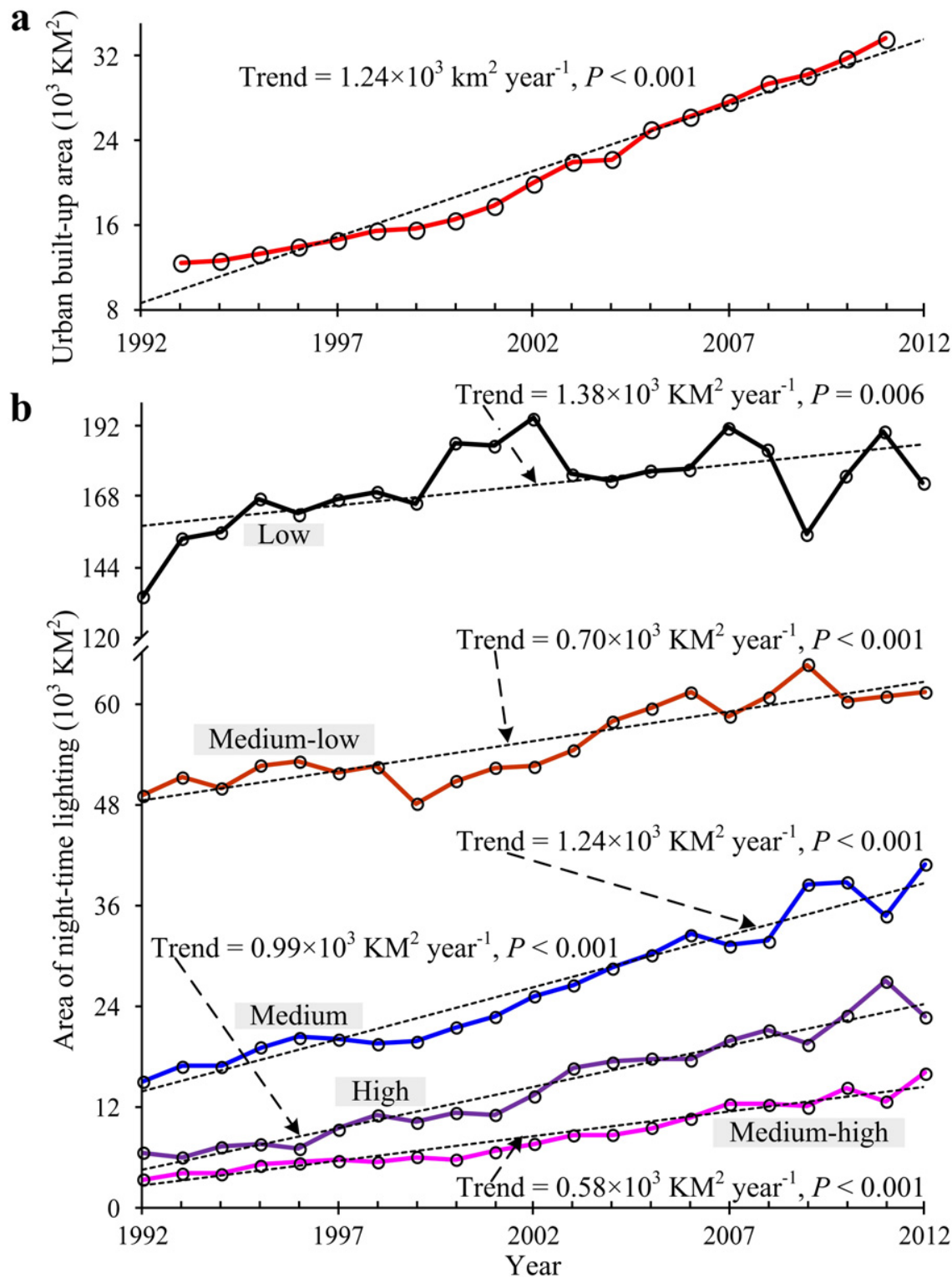

Fig. 5. Long-term trends in a) urban built-up areas and b) different types of urban night-time lighting areas across 274 China's cities from 1992 to 2012 at the country level.

Zhangzhou City, Hengshui City and Kaifeng City, low lighting areas are found to significantly decline while other four types of lighting areas commonly show a significant growth. These medium cities usually experienced a slow development within limited spatial extents in which reduced dim lighting areas were likely related to increased suburban areas. Beijing City, Shanghai City and Hanzhou City show significant trends towards rapidly decreased low and medium-low lighting areas following by high increases in medium and intense lighting areas of urban land. This implies a rapid urbanization process in these large and extra-large cities in which markedly expanded intense night lighting areas are usually associated with rapid development from initial urbanrural transition to urbanized areas.

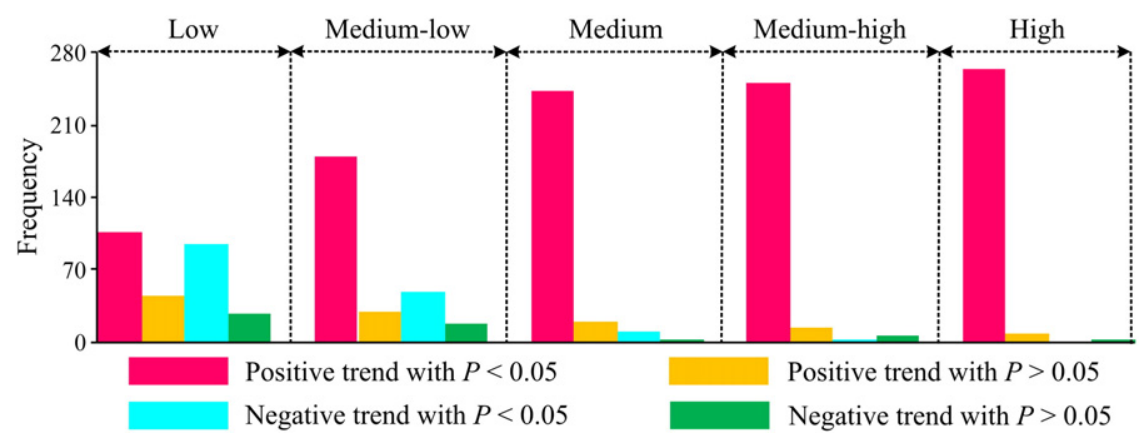

Fig. 6. Statistical distributions of 274 China's cities for linear trends (1992-2012) in different types of night-time lighted areas. 


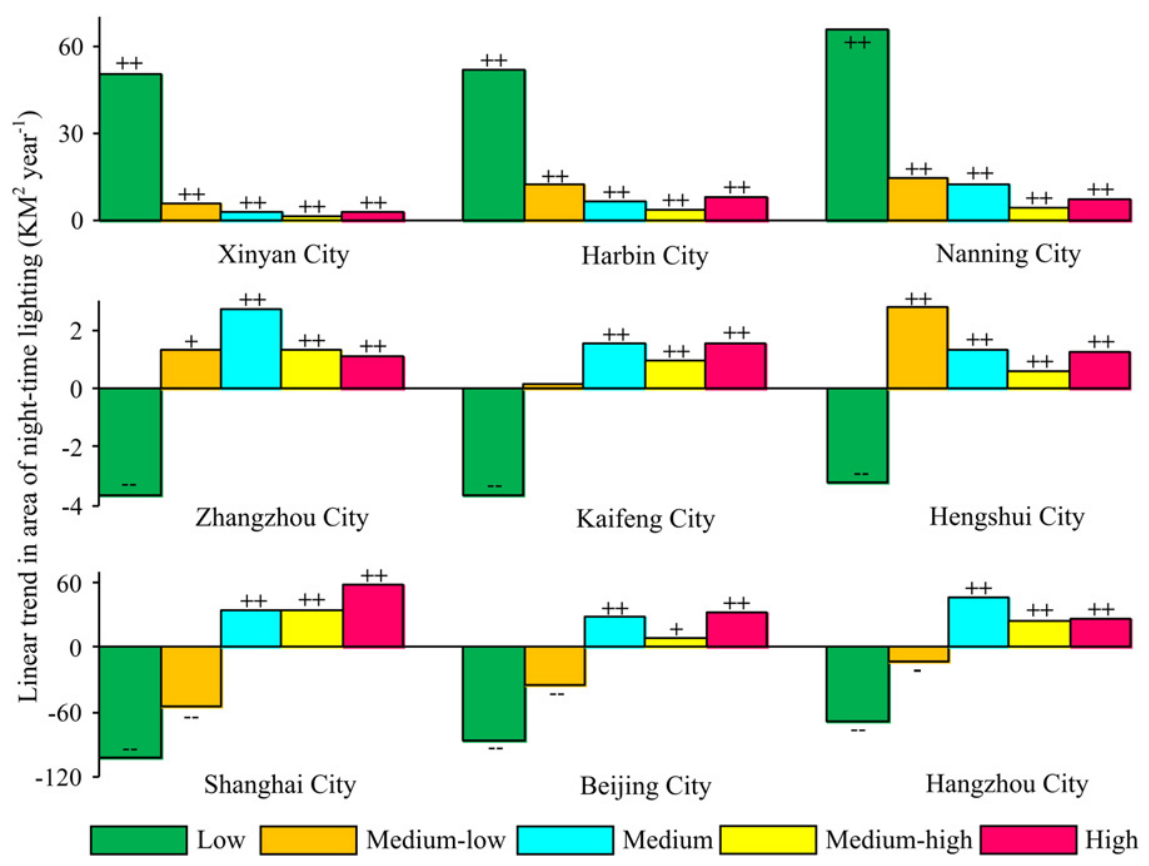

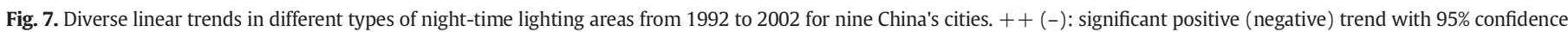
interval. $+(-)$ : marginally significant positive (negative) trend with $90 \%$ confidence interval.

\subsection{Temporal transitions of night-time lighting types}

Long-term changes and their differences in nocturnal lighted areas with different brightness magnitudes depict quantitative trends and variations of urban sub-regions with various socioeconomic activities during development at both country and local scales. Temporal transitions in different types of night-time lighted area may further characterize the footprints of urban land dynamics in terms of artificial nighttime light emissions. Table 2 presents a country-level statistical summary of pixel-based transitions in five types of night-time lighting areas between the year of 1992, 2002 and 2012 across 274 China's cities. This table suggests that $38.6 \%$ of areas with high night-time lighting in 2012 were sourced from the dim and medium-low lighting areas in 1992 (mostly located in small and medium cities). Comparably, medium and medium-high lighting areas in 1992 contributed nearly 38.7\% of the urban high lighting areas in 2012 (mostly contained in large and extra-large cities). $21.2 \%$ of high night-time lighting areas primarily remained consistent between the year of 1992 and 2012. For the medium-high night-time lighting areas in 2012, 23.8\%, 51.3\% and $20.0 \%$ of them were derived from low, medium-low and medium night-time lighting areas in 1992, respectively. Meanwhile, only 1.4\% of medium-high night-time lighting areas seem to persist unchanged. These results could indicate a rapid urban process occurred in suburban and rural-urban transition zones over the past 21 years. Furthermore, most of low and medium-low night-time lighting areas in 1992 tend to gradually develop into medium lighting areas in 2012.
At the city and region level, pixel based transitions between different types of night-time lighting areas between the year of 1992, 2002 and 2012 for three of China's extra-large cities and metropolitan regions including Beijing City, Shanghai City and Pearl River Delta are summarized in Tables 3, 4 and 5, respectively. Although these cities have commonly experienced the fastest and the most remarkable urbanization with the fastest economic growth in China since the 1990s, temporal transitions of different types of night-time lighting areas still exhibit some differences between them. The transition initially starting with mediumlow night-time lighting in 1992 accounts for $47.6 \%$ of high night-time lighting areas in 2012 in Beijing City. The proportion of such a transition decreases to 33.6\% and 16.7\% in Shanghai City and Pearl River Delta, respectively. Medium night-time lighting areas in 1992 contributed $20.8 \%$ of high night-time lighting areas in 2012 in Beijing City. This proportion increases to $24.6 \%$ in Shanghai City and 34.0\% in Pearl River Delta. Pearl River Delta shows a higher proportion of the transition from mediumhigh to high night-time lighting areas (16.5\%) than that in Beijing City (7.1\%) and Shanghai City (7.9\%). The proportion of unchanged high night-time lighting areas between 1992, 2002 and 2012 is 24.5\%, 29.3\% and 32.2\% for Beijing City, Shanghai City and Pearl River Delta, respectively. The medium-high night-time lighting areas in 2012 are mainly derived from the type of medium-low night-time lighting areas in 1992 across these three regions. Pearl River Delta, however, shows $31.6 \%$ of medium-high night-time lighting areas converted from the type of medium night-time lighting areas in 1992 while the proportion is only $8.6 \%$ and $3.8 \%$ for Shanghai City and Beijing City,

Table 2

Summary of pixel-based transitions of night-time lighting types between 1992, 2002 and 2012 across 274 China's cities.

\begin{tabular}{|c|c|c|c|c|c|c|c|c|c|c|c|c|c|c|c|c|c|c|c|c|c|c|}
\hline \multicolumn{2}{|l|}{1992} & \multirow{2}{*}{$\frac{\mathrm{L}}{\mathrm{L}}$} & \multirow{2}{*}{$\frac{\mathrm{L}}{\mathrm{ML}}$} & \multirow{2}{*}{$\frac{\mathrm{L}}{\mathrm{M}}$} & \multirow{2}{*}{$\frac{\mathrm{L}}{\mathrm{MH}}$} & \multirow{2}{*}{$\frac{\mathrm{L}}{\mathrm{H}}$} & \multirow{2}{*}{$\frac{\mathrm{ML}}{\mathrm{L}}$} & \multirow{2}{*}{$\frac{\mathrm{ML}}{\mathrm{ML}}$} & \multirow{2}{*}{$\frac{\mathrm{ML}}{\mathrm{M}}$} & \multirow{2}{*}{$\frac{\mathrm{ML}}{\mathrm{MH}}$} & \multirow{2}{*}{$\frac{\mathrm{ML}}{\mathrm{H}}$} & \multirow{2}{*}{$\frac{\mathrm{M}}{\mathrm{ML}}$} & \multirow{2}{*}{$\frac{M}{M}$} & \multirow{2}{*}{$\frac{\mathrm{M}}{\mathrm{MH}}$} & \multirow{2}{*}{$\frac{M}{H}$} & \multirow{2}{*}{$\frac{\mathrm{MH}}{\mathrm{M}}$} & \multirow{2}{*}{$\frac{\mathrm{MH}}{\mathrm{MH}}$} & \multirow{2}{*}{$\frac{\mathrm{MH}}{\mathrm{H}}$} & \multirow{2}{*}{$\frac{\mathrm{H}}{\mathrm{M}}$} & \multirow{2}{*}{$\frac{\mathrm{H}}{\mathrm{MH}}$} & \multirow{2}{*}{$\frac{\mathrm{H}}{\mathrm{H}}$} & \multirow[t]{2}{*}{ Total } \\
\hline 2002 & & & & & & & & & & & & & & & & & & & & & & \\
\hline 2012 & $\mathrm{~L}$ & $\mathrm{~N} / \mathrm{A}$ & 1985 & - & - & - & 3065 & 957 & - & - & - & - & - & - & - & - & - & - & - & - & - & $\mathrm{N} / \mathrm{A}$ \\
\hline & ML & 55,487 & 17,676 & 255 & - & - & 4238 & 11,514 & 570 & - & - & 599 & 121 & - & - & - & - & - & - & - & - & 90,460 \\
\hline & $\mathrm{M}$ & 9886 & 16,379 & 2509 & - & - & 816 & 16,046 & 8267 & 309 & - & 1182 & 2722 & 267 & - & 182 & - & - & - & - & - & 58,565 \\
\hline & $\mathrm{MH}$ & 937 & 3054 & 1535 & 124 & - & - & 3830 & 6687 & 1365 & 299 & 230 & 2713 & 1335 & 469 & 264 & 325 & 188 & 102 & 152 & 121 & 23,730 \\
\hline & $\mathrm{H}$ & 342 & 1209 & 1063 & 202 & - & - & 2031 & 6041 & 3116 & 2530 & 103 & 2879 & 3290 & 5751 & 275 & 828 & 3420 & 121 & 500 & 9048 & 42,749 \\
\hline
\end{tabular}

L: low, ML: medium low, M: medium, MH: medium-high, H: high. N/A: no data available. -: excluded transition with $<100$ pixels for simplicity. 
Table 3

Summary of pixel-based transitions of night-time lighting types between 1992, 2002 and 2012 for Beijing City.

\begin{tabular}{|c|c|c|c|c|c|c|c|c|c|c|c|c|c|c|c|c|c|c|c|c|c|c|}
\hline 1992 & & $\mathrm{~L}$ & $\mathrm{~L}$ & $\mathrm{~L}$ & $\mathrm{~L}$ & $\mathrm{~L}$ & ML & ML & ML & ML & ML & M & M & $\mathrm{M}$ & M & $\mathrm{MH}$ & MH & $\mathrm{MH}$ & $\mathrm{H}$ & $\mathrm{H}$ & $\mathrm{H}$ & Total \\
\hline 2002 & & $\mathrm{~L}$ & ML & M & $\mathrm{MH}$ & $\mathrm{H}$ & $\mathrm{L}$ & ML & M & $\mathrm{MH}$ & $\mathrm{H}$ & ML & M & $\mathrm{MH}$ & $\mathrm{H}$ & M & $\mathrm{MH}$ & $\mathrm{H}$ & M & MH & $\mathrm{H}$ & \\
\hline \multirow[t]{5}{*}{2012} & $\mathrm{~L}$ & $\mathrm{~N} / \mathrm{A}$ & 167 & - & - & - & 60 & 34 & - & - & - & - & - & - & - & - & - & - & - & - & - & $\mathrm{N} / \mathrm{A}$ \\
\hline & ML & 859 & 1559 & 22 & - & - & 15 & 764 & 20 & - & - & 18 & - & - & - & - & - & - & - & - & - & 3257 \\
\hline & M & - & 594 & 38 & - & - & - & 838 & 266 & - & - & - & 22 & - & - & - & - & - & - & - & - & 1758 \\
\hline & $\mathrm{MH}$ & - & 32 & 10 & - & - & - & 171 & 342 & - & - & - & 22 & - & - & - & - & - & - & - & - & 577 \\
\hline & $\mathrm{H}$ & - & - & - & - & - & - & 75 & 829 & 322 & 420 & - & 51 & 127 & 541 & - & 12 & 235 & - & - & 846 & 3458 \\
\hline
\end{tabular}

L: low, ML: medium low, M: medium, MH: medium-high, H: high. N/A: no data available. -: excluded transition with $<10$ pixels for simplicity.

respectively. All the above results likely demonstrate differences among the three regions. Specifically, (1) Beijing City has relatively higher enhancement in high night-time lighting areas mostly converted from medium-high lighting areas than Shanghai City and Pearl River Delta; (2) Pearl River Delta show a more rapid increase in medium-high lighting areas than Beijing City and Shanghai City; and (3) Shanghai City show a relatively balanced development in dense and medium nighttime lighting areas from 1992 to 2012.

\subsection{Spatial patterns of night-time lighting type transitions}

The urban expansion process is generally concurrent with the spatial changes in night-time light emissions. The spatial evolution of the magnitude of artificial night-time lighting therefore could potentially depict the trajectory of urban development. Fig. 8 presents the spatial patterns of pixel based type transitions of night-time lights between the year of 1992, 2002 and 2012 for several of China's cities. Overall, transitions of night-time lighting types show conspicuous spatial patterns composed of the geographical expansion of successively increased artificial night-time brightness over time around the urban central area showing consistently high night-time lighting. Type transitions between the medium and high night-time light emissions are normally located close to the central area of urban. Meanwhile, type transitions from low to medium night-time lighting likely occur in regions away from the urban central area. Thus, the spatio-temporal patterns in night-time lighting type transitions could show both spatially and temporally continuous urban development processes followed by gradually intensified human activities which geographically diffuse from the central region to the periphery of urban areas. The expansion process of the urban central area typically associated with high density impervious surface and human activity is notably linked to the spatially regular transition of night-time lighted areas with different emission brightness: while preceding medium-level night-time lighting areas became the highly lit areas, their adjacent areas normally with initial low-level night-time brightness simultaneously developed into medium-level lighting areas which could potentially become high brightness areas in the next stage. In some cities, the terrain and coastline could limit the spatial expansions of urban development, for instance, in Lanzhou City, Dalian City and Qingdao City. At the region scale, with continuously spatial expansion of increasingly high brightness areas in individual cites, whole metropolitan areas (e.g. Yangtze River Delta and Pearl River Delta) are gradually connected by artificial night-time lighting.

To examine whether the spatial expansions of increased night-time light brightness are geographically aligned with the rural-urban gradients, we have statistically tested the significance of differences between the geographical distances of pixel to the center point of urban among different transition types of night-time lights during the period of 1992-2002-2012 in several cities (Fig. 9). A two-sample $t$-test shows that, except for the transition of M-MH-H and ML-M-H in Beijing City and Shanghai City, most type transitions of night-time lighting show marked differences in the spatial distance of pixels to the center point of the corresponding city at the $95 \%$ confidence interval. Moreover, as shown in Fig. 9, all cities uniformly exhibit an increased median value of geographical distances from the stable high night-time lighting transition (H-H-H) to the low-medium level transition (L-ML-M), through the medium-high level transitions: M-MH-H, ML-M-H and M-MH-H. This result further indicates that intensifying anthropogenic nighttime lights, typically associated with escalating human population and socioeconomic activity, are geographically expanding along with the rural-urban gradients during the urbanization process.

Table 4

Summary of pixel-based transitions of night-time lighting types between 1992, 2002 and 2012 for Shanghai City.

\begin{tabular}{|c|c|c|c|c|c|c|c|c|c|c|c|c|c|c|c|c|c|c|c|c|c|c|}
\hline 1992 & & $\mathrm{~L}$ & $\mathrm{~L}$ & $\mathrm{~L}$ & $\mathrm{~L}$ & $\mathrm{~L}$ & ML & ML & ML & ML & ML & M & M & M & M & $\mathrm{MH}$ & $\mathrm{MH}$ & $\mathrm{MH}$ & $\mathrm{H}$ & $\mathrm{H}$ & $\mathrm{H}$ & Total \\
\hline 2002 & & $\mathrm{~L}$ & ML & M & $\mathrm{MH}$ & $\mathrm{H}$ & $\mathrm{L}$ & ML & M & $\mathrm{MH}$ & $\mathrm{H}$ & ML & M & $\mathrm{MH}$ & $\mathrm{H}$ & M & $\mathrm{MH}$ & $\mathrm{H}$ & M & $\mathrm{MH}$ & $\mathrm{H}$ & \\
\hline \multirow[t]{5}{*}{2012} & $\mathrm{~L}$ & $\mathrm{~N} / \mathrm{A}$ & 12 & - & - & - & 10 & - & - & - & - & - & - & - & - & - & - & - & - & - & - & N/A \\
\hline & ML & 579 & 270 & 14 & - & - & 57 & 111 & 26 & - & - & - & - & - & - & - & - & - & - & - & - & 1057 \\
\hline & $\mathrm{M}$ & 411 & 744 & 88 & - & - & 11 & 442 & 187 & - & - & - & 15 & - & - & - & - & - & - & - & - & 1898 \\
\hline & MH & 77 & 148 & 144 & - & - & - & 207 & 532 & 176 & - & - & 71 & 52 & - & - & 16 & - & - & - & - & 1423 \\
\hline & $\mathrm{H}$ & - & - & 48 & 41 & 25 & - & 21 & 169 & 404 & 252 & - & 20 & 162 & 437 & - & 20 & 180 & - & - & 737 & 2516 \\
\hline
\end{tabular}

L: low, ML: medium low, M: medium, MH: medium-high, H: high. N/A: no data available. -: excluded transition with $<10$ pixels for simplicity.

Table 5

Summary of pixel-based transitions of night-time lighting types between 1992, 2002 and 2012 for Pearl River Delta.

\begin{tabular}{|c|c|c|c|c|c|c|c|c|c|c|c|c|c|c|c|c|c|c|c|c|c|c|}
\hline 1992 & & $\mathrm{~L}$ & $\mathrm{~L}$ & $\mathrm{~L}$ & $\mathrm{~L}$ & $\mathrm{~L}$ & ML & ML & ML & ML & ML & M & M & M & M & $\mathrm{MH}$ & $\mathrm{MH}$ & $\mathrm{MH}$ & $\mathrm{H}$ & $\mathrm{H}$ & $\mathrm{H}$ & Total \\
\hline 2002 & & $\mathrm{~L}$ & ML & M & $\mathrm{MH}$ & $\mathrm{H}$ & $\mathrm{L}$ & ML & M & $\mathrm{MH}$ & $\mathrm{H}$ & ML & M & $\mathrm{MH}$ & $\mathrm{H}$ & M & $\mathrm{MH}$ & $\mathrm{H}$ & M & $\mathrm{MH}$ & $\mathrm{H}$ & \\
\hline \multirow[t]{5}{*}{2012} & $\mathrm{~L}$ & N/A & 50 & - & - & - & 18 & - & - & - & - & - & - & - & - & - & - & - & - & - & - & $\mathrm{N} / \mathrm{A}$ \\
\hline & ML & 3084 & 1708 & 15 & - & - & 159 & 453 & 16 & - & - & - & - & - & - & - & - & - & - & - & - & 5435 \\
\hline & $\mathrm{M}$ & 253 & 1803 & 612 & 17 & - & 21 & 1621 & 2286 & 153 & 16 & 68 & 440 & 83 & 17 & 40 & - & - & 10 & - & - & 7440 \\
\hline & $\mathrm{MH}$ & - & 32 & 113 & 32 & - & - & 80 & 1160 & 539 & 189 & - & 410 & 448 & 230 & 31 & 68 & 60 & - & 20 & 31 & 3443 \\
\hline & $\mathrm{H}$ & - & - & 16 & - & 16 & - & - & 200 & 282 & 365 & - & 133 & 537 & 1053 & 19 & 109 & 707 & - & 53 & 1577 & 5067 \\
\hline
\end{tabular}

L: low, ML: medium low, M: medium, MH: medium-high, H: high. N/A: no data available. -: excluded transition with $<10$ pixels for simplicity. 

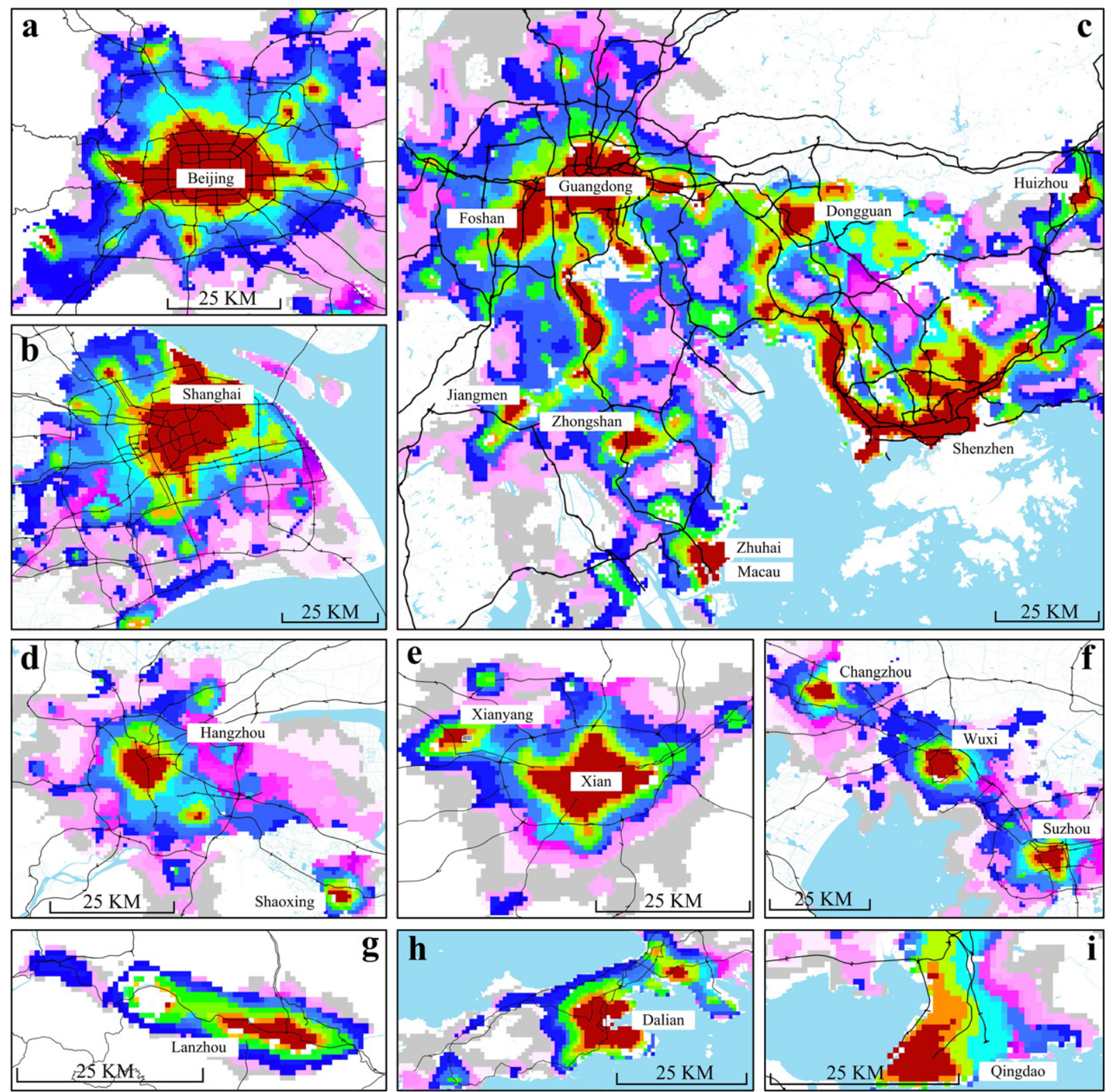

The type transition between the year of 1992-2002-2012. L: Low, ML: Medium-low, M: Medium, MH: Medium-high, H: High

\begin{tabular}{|l|l|l|l|l|l|l|l|l|}
\hline L-L-ML & L-ML-M & L-M-H & ML-ML-M & ML-M-H & M-ML-H & M-MH-H & MH-H-H \\
\hline L-L-M & L-ML-MH & L-MH-MH & ML-ML-MH & ML-MH-MH & M-M-M & M-H-H & H-H-H \\
\hline L-L-MH & L-ML-H & L-MH-H & ML-ML-H & ML-MH-H & M-M-MH & MH-M-H & Water \\
\hline L-L-H & L-M-M & L-H-H & ML-M-M & ML-H-H & M-M-H & MH-MH-MH & MH \\
\hline L-ML-ML & L-M-MH & ML-ML-ML & ML-M-MH & M-ML-MH & M-MH-MH & MH-MH-H & Major roads
\end{tabular}

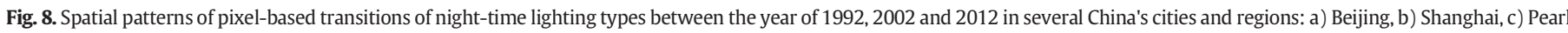

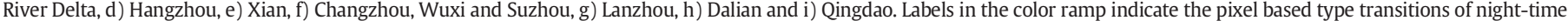
lights between the years of 1992, 2002 and 2012.

In addition, Small and Elvidge (2011, 2013) used empirical orthogonal function (EOF) analysis to map spatial and temporal changes in night-time lights. Comparisons of the projection filtered tri-temporal night-time light composites (see Fig. 3 in Small and Elvidge's paper, 2011) with the local scale quadratic curve based partitioning results (Fig. 8(c)) in the Pearl River Delta region indicate that two methods could produce similar results in characterizing the spatio-temporal patterns of night-time light dynamics. EOF based approach quantitatively outlines the spatial trajectories of the night-time lighted areas over time; our method may further depict spatio-temporal transitions of different night-time light brightness in association with urban development processes.

\section{Conclusions}

Satellite data of anthropogenic lighting emissions at night provide observationally based measures for studying human settlement development and socioeconomic activity in terms of urban sprawl, demographic dynamics, energy consumption and gross regional production in a timely and spatially explicit manner. Most previous research efforts 

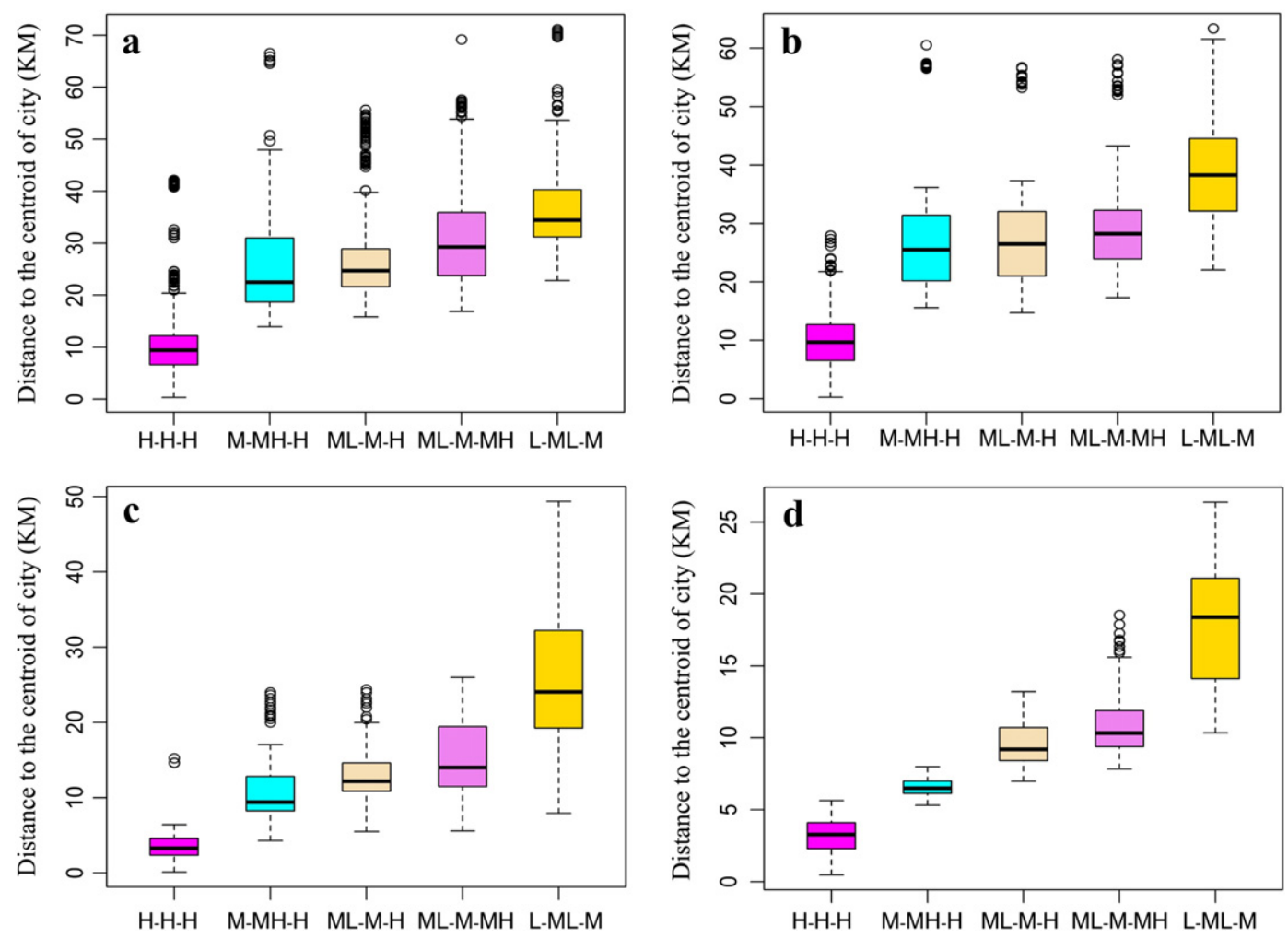

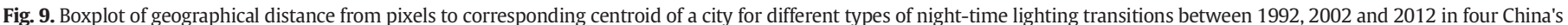
cities: a) Beijing, b) Shanghai, c) Hangzhou and d) Wuxi. The meanings of text labels are consistent with Fig. 8.

have investigated the overall quantitative responses of anthropogenic night-time brightness to demographic and socioeconomic dynamics at the regional and country levels (Elvidge, Hsu, Baugh, \& Ghosh, 2014; Elvidge et al., 2009; Ghosh et al., 2010; Sutton and Costanza, 2002; Zhang and Seto, 2011). Owing to the effects of overglow and shrink of night-time light signals, lighted areas in satellite imagery usually differ from the spatial extent of urban impervious surfaces (Small et al., 2011). Moreover, both the saturation effect of DMSP/OLS imagery and diversity in urban development forms largely limit the utility of empirical threshold-based measures for urbanized areas and internal variations of urban development. In this study, we provided an approach to partition DMSP/OLS imagery into several sub-regions potentially associated with various human activities and urbanization levels at the urban scale. Our method is based upon the quadratic relationship between the pixel-level night-time light intensity and the spatial gradient of brightness which explicitly depicts the spatial fluctuations in the magnitude of artificial night-time lighting across urban-rural region. The statistical significance of the quadratic relationship between the pixel-level NTL and $B G$ and classified night-time light imagery have been validated in 271 China's prefectural level cities and municipalities for the period of 1992-2012. The practical advantages of the local scale quadratic curve based partition method are that (1) it explicitly takes into account the locally spatial variations of night-time light signals along with the urban-rural gradients; thus (2) this approach can quantitatively identify the spatial extents for variant types of night-time brightness typically connected to different population and socioeconomic activities in a given region; and (3) it allows us to investigate not only the longterm trends in different magnitudes of artificial night-time lighting but the spatial dynamics and transitions of night-time lights patterns in relation to the urbanization process.

Based upon statistically fitted yearly quadratic curves at the urban scale, we spatially subdivided the DMSP/OLS image of a given city into five sub-areas with various magnitudes of artificial night-time light emissions: low, medium-low, medium, medium-high and high nighttime lighting areas which could be typically connected to the urban sub-regions experiencing different demographic and socioeconomic activities. Particularly, urban-level intense night-time lighting areas (including high and the sum of medium-high and high) are very close to the corresponding statistical urban built-up areas with intensified human activity. At the country level, five types of night lighting regions commonly show significant trends toward increasing lighting areas at night with the rapid urbanization across 271 China's cities from 1992 to 2012. At the city level, however, various trends in five types of night-time lighting areas over time have been found in individual cities potentially associated with different urban forms and development stages. Generally, significantly enhanced medium and intense nighttime lighting areas occur in almost all Chinese cities while synchronous increases in low and medium-low night lighting areas are observed in small and medium cites and decreased low and medium-low artificial lighting areas at night are found in most large and extra-large cities.

The quantitative transition between different types of night-time lighting areas over time could further reveal the differences in urban development in terms of human activity. For instance, although Beijing City, Shanghai City and Pearl River Delta have generally experienced the fastest urbanization processes in China since the 1990s, our results indicate that high density night-time lighting areas in Beijing City show faster growth and a greater proportion of conversion starting from medium-high night-time lighting areas than Shanghai City and Pearl River Delta between the year of 1992 and 2012. Meanwhile, Pearl River Delta has a faster development in medium-high night-time lighting areas and higher proportion of transition starting from medium night-time lighting areas than Beijing City and Shanghai City. These results could imply that Beijing City and Pearl River Delta have faster development in the urbanized areas with high density human activity and the periphery of the urban central area with increasingly strong socioeconomic activity than others over the past 21 years, respectively. Furthermore, the spatial patterns of type transition in night-time lighting areas clearly show that gradually increasing artificial lighting at night is geographically diffusing along with the rural-urban gradients following the urban expansion. 
In addition, recently composed night-time light data derived from the Visible Infrared Imaging Radiometer Suite (VIIRS) instrument aboard the NASA-NOAA Suomi National Polar orbiting Partnership satellite, provided by NOAA's National Geophysical Data Center, shows much clearer measures of anthropogenic night-time light emissions at the Earth's surface with zero moonlight and without the saturation limit (Miller et al., 2012). The notable advantages of VIIRS imagery are higher spatial resolution (15 arc-second), lower detection limits and more sensitive to socioeconomic activity than DMSP/OLS data (Baugh, Hsu, Elvidge, \& Zhizhin, 2013). Further studies are needed to investigate the applicability of the local scale quadratic curve based partition method in order to improve the application of VIIRS data to characterizing the spatio-temporal patterns of human activity and urbanization dynamics through artificial night-time lighting signals.

\section{Acknowledgments}

This research has been funded by the National Natural Science Foundation of China (No. 41371379) and the Major State Basic Research Development Program of China (No. 2015CB954101). We would like to gratefully thank the anonymous reviewers for their insightful and helpful comments to improve the manuscript.

\section{References}

Baugh, K., Hsu, F. C., Elvidge, C. D., \& Zhizhin, M. (2013). Nighttime lights compositing using the VIIRS day-night band: Preliminary results. Proceedings of the Asia-Pacific Advanced, Network, 55, 70-86.

Burrough, P. A., \& McDonell, R. A. (1998). Principles of geographical information systems. New York: Oxford University Press.

Chen, X., \& Nordhaus, W. D. (2011). Using luminosity data as a proxy for economic statistics. Proceedings of the National Academy of Sciences of the United States of America, $108,8589-8594$.

Cohen, B. (2006). Urbanization in developing countries: Current trends, future projections, and key challenges for sustainability. Technology in Society, 28, 63-80.

Croft, T. A. (1973). Burning waste gas in oil fields. Nature, 245, 375-376.

Doll, C. N. H., Muller, J. P., \& Elvidge, C. D. (2000). Night-time imagery as a tool for global mapping of socioeconomic parameters and greenhouse gas emissions. Ambio, 29 $157-162$.

Doll, C. N. H., Muller, J. P., \& Morley, J. G. (2006). Mapping regional economic activity from night-time light satellite imagery. Ecological Economics, 57, 75-92.

Elvidge, C. D., Baugh, K. E., Anderson, S. J., Sutton, P. C., \& Ghosh, T. (2012). The Night Light Development Index (NLDI): A spatially explicit measure of human development from satellite. Social Geography, 7, 23-35.

Elvidge, C. D., Baugh, K. E., Dietz, J. B., Bland, T., Sutton, P. C., \& Kroehl, H. W. (1999). Radiance calibration of DMSP-OLS low light imaging data of human settlements. Remote Sensing of Environment, 68, 77-88.

Elvidge, C. D., Baugh, K. E., Kihn, E. A., Kroehl, H. W., \& Davis, E. R. (1997). Mapping city lights with nighttime data from the DMSP Operational Linescan System. Photogrammetric Engineering \& Remote Sensing, 63, 727-734.
Elvidge, C. D., Hsu, F. C., Baugh, K. E., \& Ghosh, T. (2014). National trends in satellite observed lighting: 1992-2012. Global urban monitoring and assessment through earth observation. CRC Press, 97-120.

Elvidge, C. D., Imhoff, M. L., Baugh, K. E., Hobson, V. R., Nelson, I., Safran, J., et al. (2001) Night-time lights of the world: 1994-1995. ISPRS Journal of Photogrammetry E Remote Sensing, 56, 81-99.

Elvidge, C. D., Ziskin, D., Baugh, K. E., Tuttle, B. T., Ghosh, T., Pack, D. W., et al. (2009). A fifteen year record of global natural gas flaring derived from satellite data. Energies, 2, 595-622.

Folke, C., Jansson, A., Larsson, J., \& Costanza, R. (1997). Ecosystem appropriation by cities Ambio, 26, 167-172.

Ghosh, T., Anderson, S. J., Elvidge, C. D., \& Sutton, P. C. (2013). Using nighttime satellite imagery as a proxy measure of human well-being. Sustainability, 5, 4988-5019.

Ghosh, T., Powell, R. L., Elvidge, C. D., Baugh, K. E., Sutton, P. C., \& Anderson, A. (2010) Shedding light on the global distribution of economic activity. The Open Geography Journal, 3, 148-161.

Imhoff, M. L., Lawrence, W. T., Stutzer, D. C., \& Elvidge, C. D. (1997). A technique for using composite DMSP-OLS “City Lights" satellite data to map urban area. Remote Sensing of Environment, 61, 361-370.

Johnson, M. P. (2001). Environmental impacts of urban sprawl: A survey of the literature and proposed research agenda. Environment and Planning A, 33, 717-735.

Miller, S. D., Mills, S. P., Elvidge, C. D., Lindsey, D. T., Lee, T. F., \& Hawkins, J. D. (2012). Suomi satellite brings to light a unique frontier of nighttime environmental sensing capabilities. Proceedings of the National Academy of Sciences of the United States of America, 109, 15706-15711.

Montgomery, M. (2008). The urban transformation of the developing world. Science, 319 761-764.

National Bureau of Statistics of China (1993-2013). Urban statistical yearbook of China Beijing: China Statistical Press.

Pugh, C. (2000). Sustainable cities in developing countries: Theory and practice at the millennium. London: Taylor \& Francis.

Shukla, V., \& Parikh, K. (1992). The environmental consequences of urban growth: Cross national perspectives on economic development, air pollution, and city size. Urban Geography, 13, 422-449.

Small, C., \& Elvidge, C. D. (2011). Mapping decadal change in anthropogenic night light. Procedia Environmental Sciences, 7, 353-358.

Small, C., \& Elvidge, C. D. (2013). Night on earth: Mapping decadal changes of anthropogenic night light in Asia. International Journal of Applied Earth Observation and Geoinformation, 22, 40-52.

Small, C., Elvidge, C. D., Balk, D., \& Montgomery, M. (2011). Spatial scaling of stable night lights. Remote Sensing of Environment, 115, 269-280.

Small, C., Pozzi, F., \& Elvidge, C. D. (2005). Spatial analysis of global urban extent from DMSP-OLS night lights. Remote Sensing of Environment, 96, 277-291.

Sutton, P. C. (2003). A scale-adjusted measure of "urban sprawl" using nighttime satellite imagery. Remote Sensing of Environment, 86, 353-369.

Sutton, P. C., \& Costanza, R. (2002). Global estimates of market and non-market values derived from nighttime satellite imagery, land cover, and ecosystem service valuation. Ecological Economics, 41, 509-527.

Sutton, P., Roberts, D., Elvidge, C., \& Baugh, K. (2001). Census from heaven: An estimate of the global human population using night-time satellite imagery. International Journal of Remote Sensing, 22, 3061-3076.

Zhang, Q., \& Seto, K. C. (2011). Mapping urbanization dynamics at regional and global scales using multi-temporal DMSP/OLS nighttime light data. Remote Sensing of Environment, 115, 2320-2329. 